\title{
Dengue Virus 2
}

National Cancer Institute

\section{Source}

National Cancer Institute. Dengue Virus 2. NCI Thesaurus. Code C112267.

The serotype labeled 2 of the viral species Dengue virus, which is a single-stranded, positive-sense RNA virus of the Flaviviridae family and Flavivirus genus, and causes dengue fever. 